\title{
Sonar and Pi Based Aid for Blind
}

\section{Kavitha Viswanathan}

Department of Electronics and

Telecommunication

Vivekanand Education Society's Institute of

Technology Mumbai, Maharashtra 400074, India

kavi1388@gmail.com

\section{Prof. Sharmila Sengupta}

Department of Computer Engineering

Vivekanand Education Society's Institute of

Technology Mumbai, Maharashtra 400074, India

sharmila.sengupta@ves.ac.in

\begin{abstract}
This paper shows evidence of using Sound Navigation and Ranging (SONAR) based system. SONAR system has widespread applications in underwater scenarios. We have focused on its use in real-time detection of an obstacle and guidance in accordance to the position of the nearest obstacle. The device designed and described in this paper aids the blind in obstacle avoidance. The device when embedded in a walking, stick makes the system portable, hassle-free, unobtrusive and unprecedented. There are several ETAs (Electronic Travel Aids) designed to make blind navigation independent of human assistance. The development of Sonar and $\mathrm{Pi}$ combination does not focus on the need of path planning instead it provides obstacle avoidance. The system requires nil preplanning making it ideal for any area and location. Some of the existing systems including GPS based system (Dhristri [1], touch and go system (hand gadget with earpiece)[2] and NAVTAR[3] focus on path planning i.e. to guide the blind from source to destination. However, the sonar-pi aid aims purely at obstacle avoidance thereby making it less bulky and increased cost-effectiveness than other systems. The designed system uses Raspberry pi 2 making it suitable for further developments.
\end{abstract}

Keywords: SONAR; obstacle avoidance; raspberry pi

\section{INTRODUCTION}

As per a survey held by TOI, India has the largest blind population. Around 15 million people are either blind or have a visual disability [4]. In addition to the existing problems of a sightless person, the inattentive architectures of India accumulate and become an integral part of the visionless individual. Thus, the engineers of today have to deal with the necessity of building ETAs.

Here, we present the prototype of a sonar-pi device based on the principle of echolocation. Echolocation uses sound waves and its echoes to determine the location, texture and density of obstacles around. Daniel Kish is one blind individual who is an example of human echolocation [5]. He uses his tongue clicks, which reflects back and gives him an estimate of the obstacle location. However not all blind humans are capable of such echolocation. For the vast majority incapable of human echolocation, sonar-pi device presented here will provide audio instructions to enable them seamless movement.

A visually impaired individual faces two major challenges in his daily routine. One is being unaware of the path he needs to take to reach from say position A to position B. Systems designed for this purpose involve GPS systems or a database with pre-loaded digital maps [1]. Another challenge is avoiding obstacles. There are several systems designed for this as well. Some include beeping when an obstacle is nearby. An aerial obstacle detection with 3D mobile devices [6] gained popularity in the recent years. It does the same thing i.e. beams when an obstacle is nearby. The camera inbuilt in the cell phone is used for detection of obstacle.

The prototype designed uses SONAR sensors to detect obstacle and using voice guidance the blind is safely navigated irrespective of his source and destination. Absence of the need to know the source and destination eliminates the dependence on GPS systems and on speech processing algorithms as well.

The economical, compact and easily available sonar sensor HC-SR04 is used in the prototype in combination with Raspberry pi two Model B. This embedded system when integrated in the walking stick will prove to be an efficient aid for the blind. 


\section{EXISTING SYSTEMS}

Earlier, we mentioned some existing navigation systems. A deeper study of these helps us visualize the drawbacks, which we need to overcome in our prototype.

NOPPA architecture [7], proposed in Finland in 2004, aimed to provide a personal navigation and information system for users of public transport. The source and destination stops were treated as user inputs while using the up-to-date internet database systems, door-to-door guidance using public transport was provided. This was an additional system overlaying the walking stick or guide dog. NOPPA depended greatly on mobile internet services. Thus, areas with lower connectivity caused the system to fail. In addition, the speech-user interface requirement made the system expensive.

TANIA (Tactile Acoustical Navigation and Information Assistant) system, developed in 2011, uses RFID technology to recognize the objects using RFID tags, also provides guidance to the user depending on the map of the environment. This implies installation of RFID tags in the area where the system is used. This pre-planning may not be a viable option always. This makes it tough to use outdoors as well.

In Drishti systems (2012), the blind carry a portable computer (Xybernaut MAIV) as a backpack, which is replaced by a cell phone in more recent proposals. This potable device contains the database of the region marked for its use. The user can give the destination as an audio input. The GPS system will guide and take the user to the destination using the shortest, obstacle-free path. One limitation of GPS-based systems is the inaccuracy in object location, which can be radially $10-15 \mathrm{~m}$ away from the returned co-ordinates. Increase in the number of visible satellites reduces this radial distance to $4-5 \mathrm{~m}$. In comparison, the accuracy using HC-SR04 (the sonar sensor used in our proposed system) can be up to $3 \mathrm{~mm}$ [9]. The GPS-based systems fail in scenarios where digital map of the region is not preloaded. A cane or guide dog is needed for short distances. The system fails to provide cautionary advice for obstacles that were not present at the time of the map loading and may have come into existence later. The dependence on battery is another drawback.

PERCEPT Based Interactive Wayfinding for Visually Impaired Users in Subways (2015) is a system suggested in California based on the recent technology of NFC tags. Similar to RFID, NFC tags have to be positioned at every station. That is entire city pre-planning is required. However, the use of NFC tags has not gained enough momentum India.

None of the devices explained above help in detection of moving obstacle. In the system that provide obstacle detection, the device beeps in vicinity of an obstacle but does not direct the user regarding the favorable direction of movement.

The drawbacks of these systems can be overcome by using the SONAR-pi based system.

\section{Proposed System}

\subsection{Proposed Block Diagram}

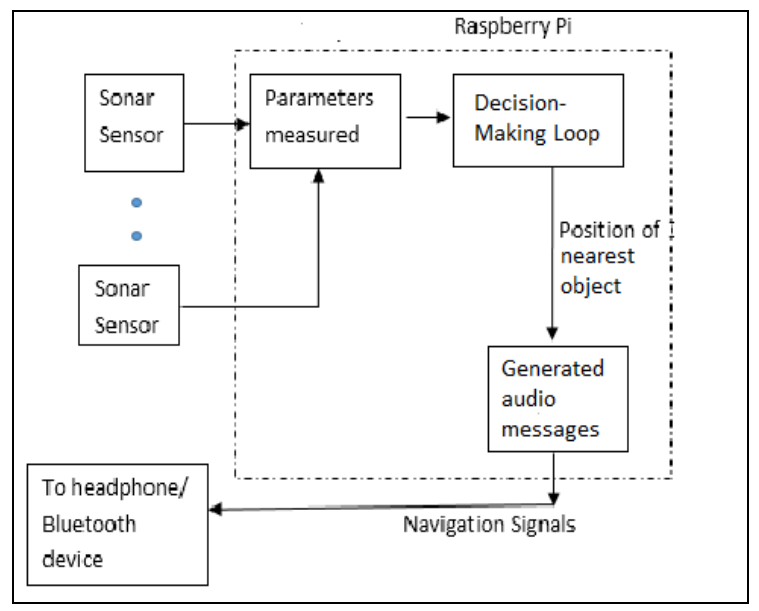

Fig1. System Block Diagram 
Navigation system for the blind can be implemented using the block diagram shown below (Fig. 2).

Fig 1 shows the block diagram of the developed system. The Sonar-pi system consists of sonar sensors, raspberry pi and headphones to give precautionary advice to the blind. We will discuss the elements in brief.

\subsection{SONAR Sensors}

In a prelude paper [11] to this system, the sonar sensor's performance was analyzed. The SONAR sensor used is HC-SR04. The graph below enables us to understand the performance of sonar sensor at different inclinations.

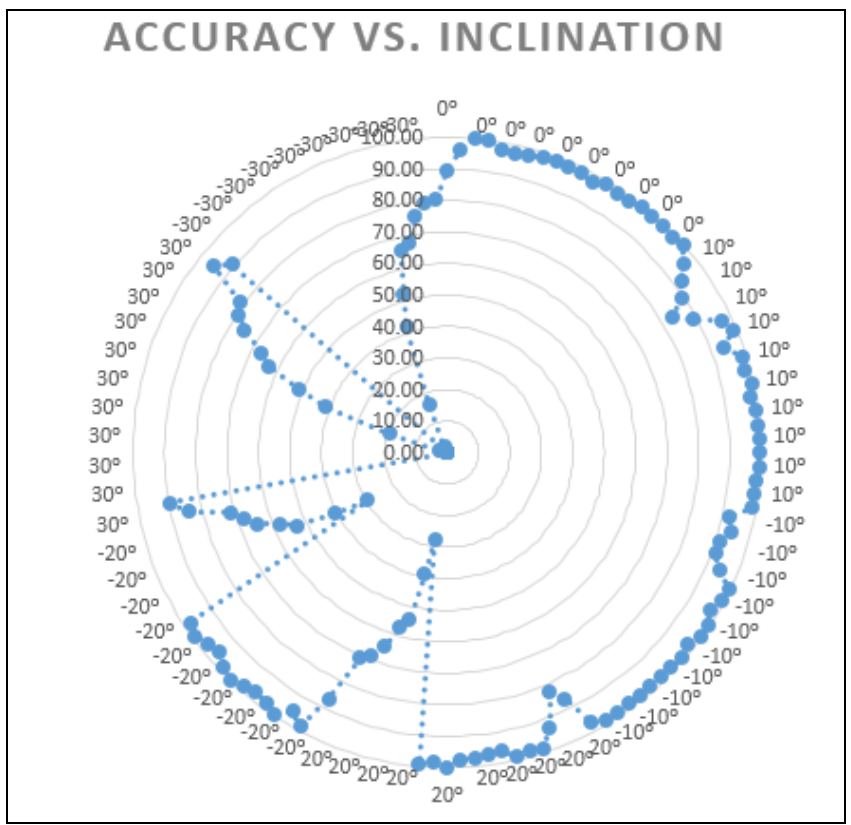

Fig2. Accuracy of sensor at different inclinations

The conclusion drawn in the previous paper, using the graph and other readings, is that a single sensor gives optimum performance at an angle of $20^{\circ}$ both clockwise and anti-clockwise directions. Thus, we have adopted three sonar sensors in our device. Each sensor is placed at an angle of $40^{\circ}$ from the adjacent one.

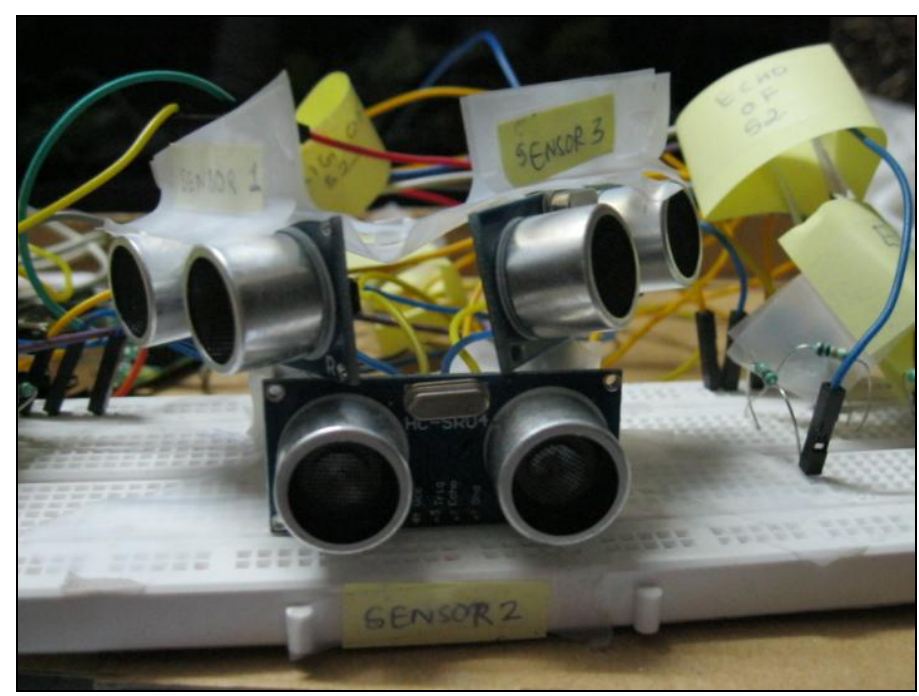

Fig3. Position of SONAR sensors

In the prototype, the sonar sensors are placed in a breadboard as shown in fig. 3. The sensors are positioned to provide minimum dead-zone. Dead-zone is that area between the sensors where an obstacle will go undetected. A sensor can detect an obstacle up to the distance of $400 \mathrm{~cm}$ with an inclination on $20^{\circ}$ horizontally and total $30^{\circ}$ vertically can be measured with reliable accuracy. However, for the safety of a visually impaired person walking, obstacles up to $200 \mathrm{~cm}$ are only considered. 


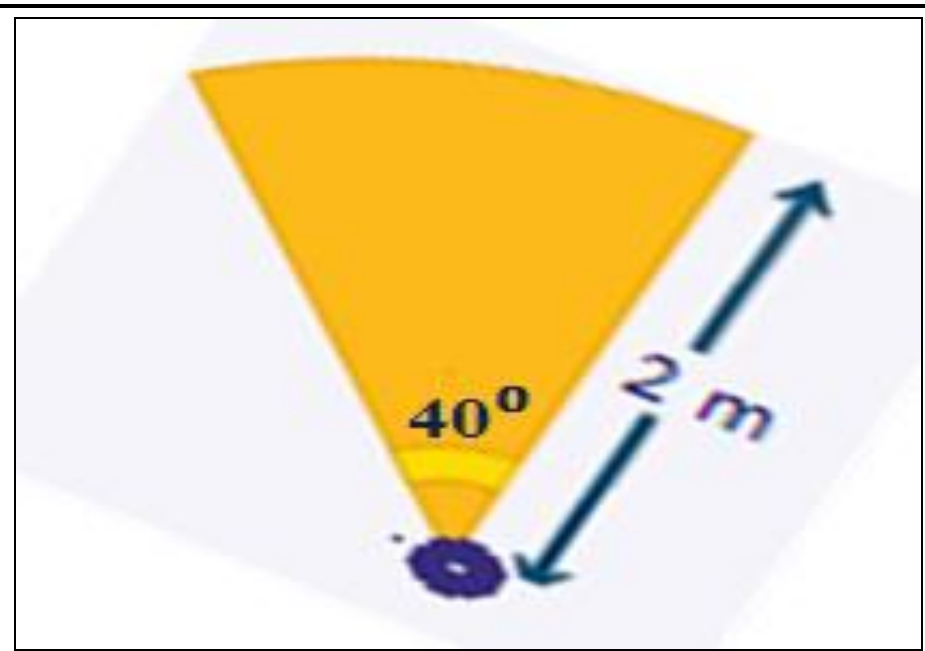

Fig4. Range of one HC-SRO4 sensor

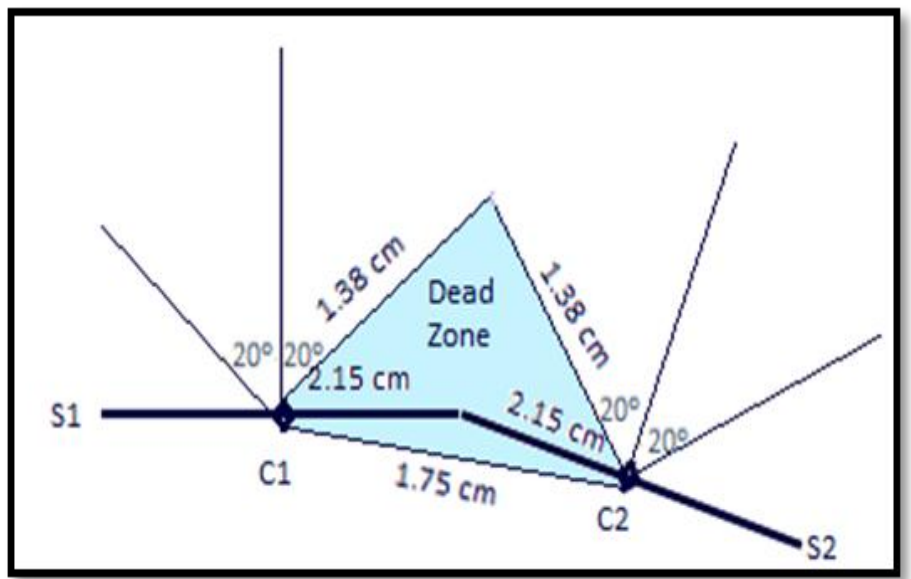

Fig5. Minimum Dead-zone Between 2 Sensors

The figure above shows the arrangement for minimum dead-zone. This is the arrangement adopted for the presented prototype.

Ideally, a walking stick height must be from the persons hand to the ground [12]. An average walking cane height is assumed $36 \mathrm{~cm}$ [13]. Thus, the device must be placed at a distance of $17-18 \mathrm{~cm}$ from the ground.

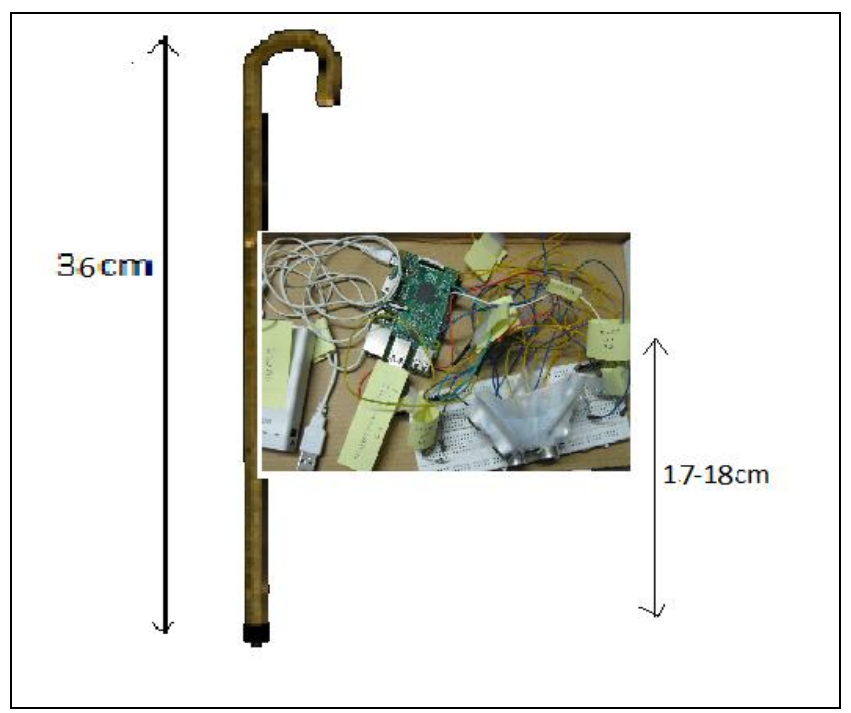

Fig6. Position of Sonar-pi device in a walking stick

Fig. 6 shows the position of the sonar-pi system in a walking stick. Vertically, an obstacle below $10^{0}$ and above $20^{\circ}$ cannot be detected. Thus, obstacles smaller than $18 \mathrm{~cm}$ at a horizontal distance of $100 \mathrm{~cm}$ will go undetected. 


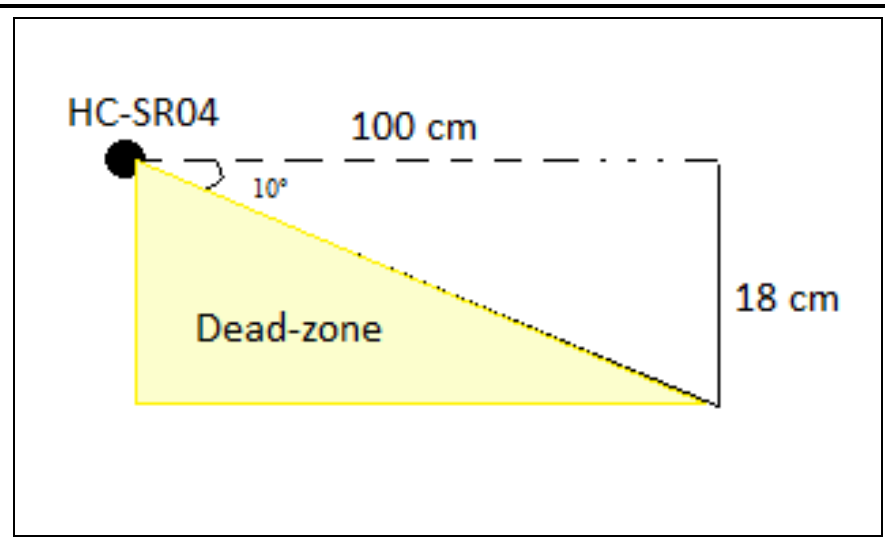

Fig7. Vertical Dead-zone

\subsection{Raspberry Pi}

The second module of our prototype is the raspberry pi 2. This system could have been developed using any other microcontroller. However, raspberry pi ensures higher processing speeds, inbuilt sound card, Broadcom card, Bluetooth and earphone ports etc. all in the size of a credit card. It has inbuilt TTS (text to speech) soft wares which may be helpful for future work.

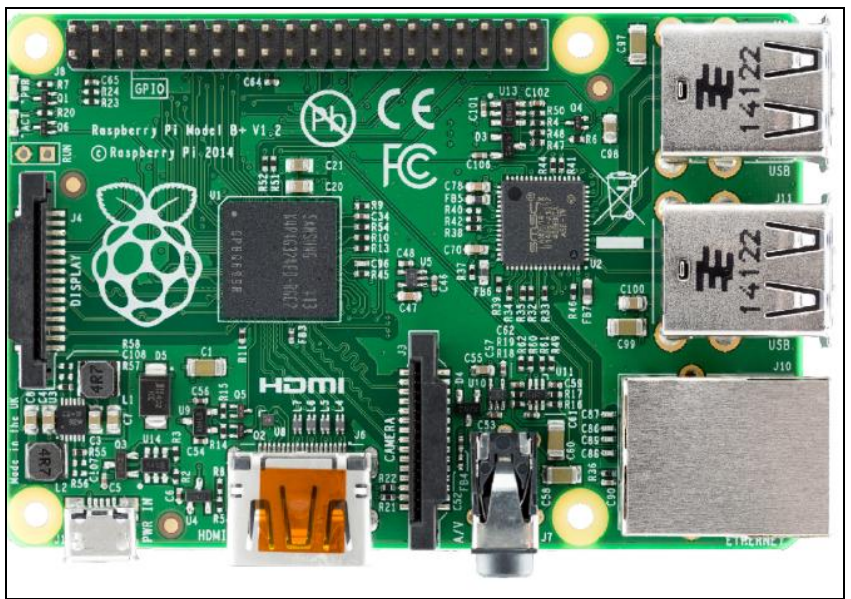

Fig8. Raspberry Pi 2

The raspberry pi is used headless in the prototype. The pi interacts with HC-SR04 using the GPIO (general-purpose input output) pins.

As stated in the previous paper, the HC-SR04 enables distance measurement using the ECHO pin. The signal from the ECHO pin acts as in input to the pi board. However, the ECHO signal is at $5 \mathrm{~V}$ whereas the GPIO pins at the pi board must be operated at $3.3 \mathrm{~V}$. To avoid damage to the pi board, the ECHO signal is sent to the pi board using a voltage divider circuit, or through a $1 \mathrm{kohm}$ resistor. Either of these methods will ensure the protection of the pi board.

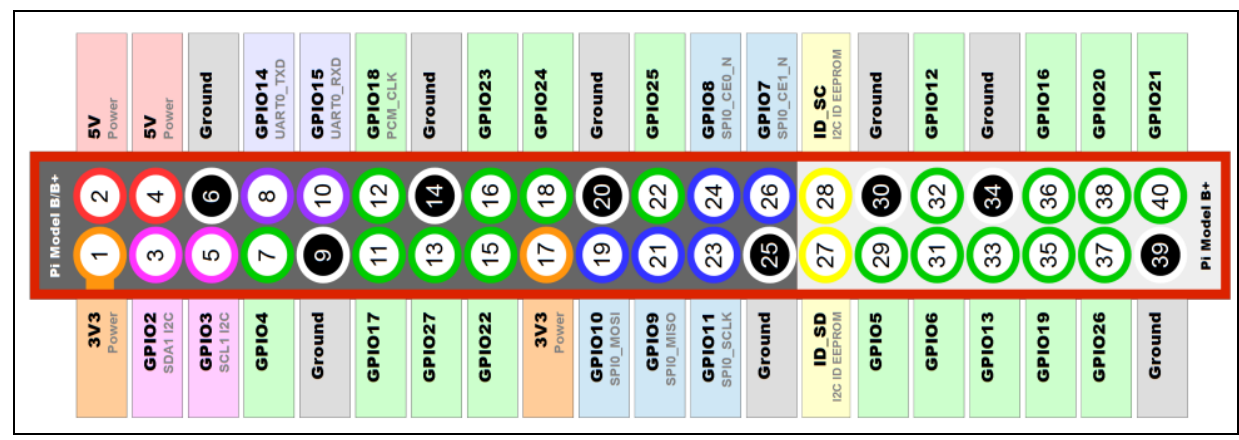

Fig9. GPIO pins

Audio messages are generated as precautionary messages to the blind using headphone. This can be replaced with a Bluetooth headphone. The audio messages are recorded with the assumption that an average human takes approx. 60 strides in a minute and has a speed of $0.3 \mathrm{~m} / \mathrm{s}$. Each step is assumed to be of $20 \mathrm{~cm}$. In reality, this will vary person to person. 


\section{IMPLEMENTATION}

In the prototype, we have used 3 sensors as shown in figure 3. The rightmost sensor is labelled as sensor 1(right facing away from the user), the middle one is labelled as sensor 2 and the leftmost one as sensor 3. In practice, more sensors can be employed for higher efficiency. The algorithm for the generation of audio messages is as shown below:

Step 1: Start

Step 2: Assign signals to GPIO pins

For sensor 1

TRIG $=$ GPIO 24

$\mathrm{ECHO}=\mathrm{GPIO} 23$

For sensor 2

TRIG $=$ GPIO 17

$\mathrm{ECHO}=\mathrm{GPIO} 22$

For sensor 3

TRIG $=$ GPIO 21

$\mathrm{ECHO}=\mathrm{GPIO} 20$

Step 3: Send a Trig pulse to sensor 2

Step 4: Check the ECHO pin for response received.

Step 5: $\mathrm{d} 2=1$ Distance $2=$ time to receive ECHO response*34300/2

Step 6: Mark this time as 1time_d2

Step 7: Repeat steps 3 to 6 for sensor 1 and sensor 3

Step 8: $\mathrm{d} 1$ and $\mathrm{d} 3$ are inclined distances. To get actual distance from these sensors:

$$
\mathrm{d} 1=\mathrm{d} 1-4.5 \sin 40-1 \& \mathrm{~d} 3=\mathrm{d} 3-4.5 \sin 40-1
$$

Step 9: if $\mathrm{d} 2>15 \mathrm{~cm}$, user is a given a message "Walk Straight"

Step 10: if $\mathrm{d} 2<15 \mathrm{~cm} \& \mathrm{~d} 1>15 \mathrm{~cm}$, user is a given a message "take left"

Step 11: if $\mathrm{d} 2<15 \mathrm{~cm} \& \mathrm{~d} 1<15 \mathrm{~cm} \& \mathrm{~d} 3>15 \mathrm{~cm}$, user is a given a message "take right"

Step 12: if $\mathrm{d} 2<15 \mathrm{~cm} \& \mathrm{~d} 1<15 \mathrm{~cm} \& \mathrm{~d} 3<15 \mathrm{~cm}$, user is a given a message "go back"

Step 13: Repeat steps 3 to 12 for 2 distance2 etc.

Step 14: Estimated time=diff in distance between 2 distance2 and 1 distance2/avg. human speed i.e. 0.3 $\mathrm{m} / \mathrm{s}$.

Step 15: Actual time=diff in time between 2time_d2 and 1time_d2

Step 16: If actual time < estimated time, moving obstacle detected and message given to user.

Step 17: Repeat steps 14 to 16 for sensor 2 and 3 for detecting moving obstacle at the left or right side.

Step 18: Repeat step 2 to 17 when $\mathrm{i} \% 10==0 ; \mathrm{i}=\mathrm{i}+1$ while $1==1$.

\section{RESULTS}

The logic used for embedding the sonar system in raspberry pi 2 is explained using the truth table given below. Here d1, d2 and d3 is for distance measured in sensor 1, 2 and 3 respectively. Here 0 implies obstacle is at a distance beyond $15 \mathrm{~cm}$ while 1 means obstacle has a value within $15 \mathrm{~cm}$. 
Table1. Results for stationary obstacles

\begin{tabular}{|c|c|c|c|c|}
\hline Sr. No. & d1 & d2 & d3 & Action \\
\hline 1. & 0 & 0 & 0 & Walk Straight \\
\hline \multirow[t]{3}{*}{ E.g. } & 222 & 300 & 245 & Walk Straight \\
\hline & 222 & 100 & 80 & Walk Straight \\
\hline & 90 & 30 & 80 & Walk Straight \\
\hline 2. & 0 & 0 & 1 & Walk Straight \\
\hline \multirow[t]{2}{*}{ E.g. } & 122 & 200 & 12 & Walk Straight \\
\hline & 150 & 50 & 12 & Walk Straight \\
\hline 3. & 1 & 0 & 0 & Walk Straight \\
\hline \multirow[t]{2}{*}{ E.g. } & 10 & 210 & 200 & Walk Straight \\
\hline & 10 & 21 & 200 & Walk Straight \\
\hline 4. & 0 & 1 & 0 & Left/Right \\
\hline E.g. & 100 & 10 & 50 & Left \\
\hline E.g. & 20 & 11 & 50 & Left \\
\hline 5. & 1 & 1 & 0 & Right \\
\hline E.g. & 12 & 10 & 25 & Right \\
\hline 6. & 1 & 1 & 1 & Go Back \\
\hline E.g. & 11 & 14 & 13 & Go Back \\
\hline
\end{tabular}

The table above summarizes the behavior of proposed system in presence of only stationary obstacles. However, in real world scenario, moving obstacles can be present such as a person walking, moving car etc.

The results obtained for moving obstacles is summarized below. The average human walking speed is assumed as $0.3 \mathrm{~m} / \mathrm{s}$.

Table2. Results for detecting moving obstacles

\begin{tabular}{|l|l|l|l|}
\hline & d1 & d2 & d3 \\
\hline $1^{\text {st }}$ value & 210 & 240 & 300 \\
\hline $2^{\text {nd }}$ value & 180 & 180 & 240 \\
\hline T_estimated & $0.1 \mathrm{sec}$ & $0.2 \mathrm{sec}$ & $0.2 \mathrm{sec}$ \\
\hline T_actual & $0.11 \mathrm{sec}$ & $0.1 \mathrm{sec}$ & $0.21 \mathrm{sec}$ \\
\hline Action & Message to user “Moving obstacle ahead” \\
\hline $1^{\text {st }}$ value & 210 & 240 & 300 \\
\hline $2^{\text {nd }}$ value & 180 & 180 & 240 \\
\hline T_estimated & $0.1 \mathrm{sec}$ & $0.2 \mathrm{sec}$ & $0.2 \mathrm{sec}$ \\
\hline T_actual & $0.11 \mathrm{sec}$ & $0.21 \mathrm{sec}$ & $0.11 \mathrm{sec}$ \\
\hline Action & Message to user “Moving obstacle to your right” & \multicolumn{2}{|l|}{} \\
\hline $1^{\text {st }}$ value & 210 & 240 & 300 \\
\hline $2^{\text {nd }}$ value & 180 & 180 & 240 \\
\hline T_estimated & $0.1 \mathrm{sec}$ & $0.2 \mathrm{sec}$ & $0.2 \mathrm{sec}$ \\
\hline T_actual & $0.06 \mathrm{sec}$ & $0.23 \mathrm{sec}$ & $0.21 \mathrm{sec}$ \\
\hline Action & Message to user “Moving obstacle to your left” \\
\hline
\end{tabular}

\section{Conclusion}

\subsection{Approximate Weight}

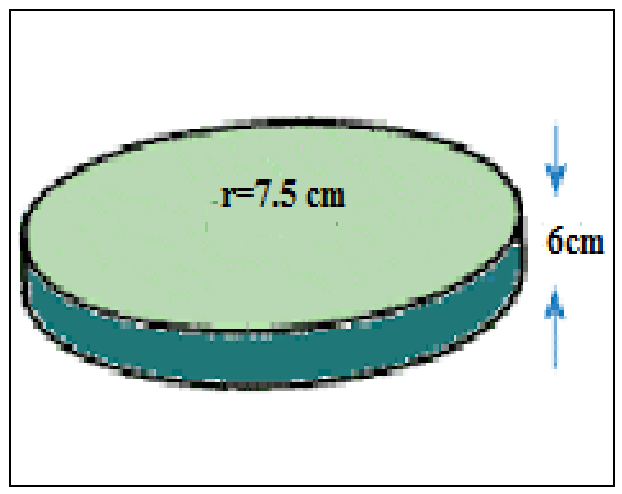

Fig10. Proposed Encasement for the device 
For embedding the device in a walking cane, the breadboard can be replaced with a PCB. The SONAR sensors can be mounted vertically on the cane or on the belt of the blind user. Using a belt, number of sensors can be can increased for increased coverage.

The individual weights of the components were taken into consideration along with their dimensions.

After careful examination, a cylindrical encasement of the following dimension is ideal for use.

The weights of the components are summarized in Table 3.

Table3. Dimension and weight of system

\begin{tabular}{|l|l|l|}
\hline Component & Dimension (cm) & Approximate weight \\
\hline HCSRO4 & $4.45 \times 2.7 \times 1.7$ & $3 \times 9=27 \mathrm{~g}$ \\
\hline PCB & $10.1 \times 7.3$ & $171 \mathrm{~g}$ \\
\hline Raspberry pi & $8.56 \times 5.398$ & $44 \mathrm{~g}$ \\
\hline Plywood & $7.5 \mathrm{~cm}$ cylinder & $0.9 \mathrm{gm} / \mathrm{cubic} \mathrm{cm}$ \\
\hline
\end{tabular}

\section{FUTURE WORK}

Using Raspberry Pi with SONAR opens up abundant future work ideas. One simple addition to the existing prototye could be enabling path planning. For this, the GPS co-ordinates can be fed on a preloaded digital map [1] or on the memory card of raspberry pi. The Raspberry pi has an inbuilt SoC Broadcom BMC2835 that enable itnernet connection in Raspberry pi. Thus the device can be used for obstacle detection as well as path planning. However, the device will fail in areas where internet connection is weak.

This paper presents the results of the prototype in an indoor environment. With appropriate setup it can be used in outdoor environment as well as the device can even detect moving obstacles like car etc. The sonar sensors can be embedded in the belt in vertical position if desired for enhancing the device's performance.

\section{REFERENCES}

[1] Abdelsalam (Sumi) Helal, Steven Edwin Moore, Balaji Ramachandran, "Drishti: An Integrated Navigation System for Visually Impaired and Disabled"- Microsoft Research under grant number 4514203-12

[2] Natalia Ponomareva, "TOUCH FEELY NAVIGATION", Yanko Design

[3] Eelke Folmer, "NAVTAR",http://brianmanzullo.com/post/77754838541/navatar-an-indoornavigation-system-for-blind

[4] http://timesofindia.indiatimes.com/india/India-has-largest-blindpopulation/articleshow/2447603.cms

[5] http://www.bbc.com/news/magazine-19524962

[6] J.M. S'aez, F. Escolano, M.A. Lozano,"Aerial obstacle detection with 3D mobile devices" IEEE 2013 Journal of Biomedical and Health Informatics.

[7] Virtanen Ari, Koskinen Sami "NOPPA ,NAVIGATION AND GUIDANCE SYSTEM FOR THE BLIND", VTT Industrial Systems

[8] Visualization and Interactive Systems Group at the University of Stuttgart, "TANIA Indoor/Outdoor Navigation System with RFID Technology"; http://www.blindnavigationinternational.org/index_en.html

[9] Product User's Manual - HCSR04 Ultrasonic Sensor

[10] Aura Ganz, James M. Schafer, Yang Tao, "PERCEPT Based Interactive Wayfinding for Visually Impaired Users in Subways", Electrical and Computer Engineering Department, University of Massachusetts

[11] Sharmila Sengupta, Kavitha Viswanathan, "Blind Navigation Proposal Using SONAR", IEEE CGVIS 2015

[12] http://www.classiccanes.co.uk/catalogue/Stick_Measurement.html

[13] http://traveltips.usatoday.com/proper-height-walking-cane-63172.html 